\title{
Multi-Modal Calibration of Surveillance Sensor Networks
}

\author{
Min Ding \\ Computer Science Department \\ George Washington University \\ Washington \\ DC 20052, USA
}

\author{
Andreas Terzis \\ Computer Science Department \\ Johns Hopkins University \\ Baltimore \\ MD 21218, USA
}

\author{
I-Jeng Wang Dennis Lucarelli \\ Applied Physics Lab \\ Johns Hopkins University \\ Laurel \\ MD 20723, USA
}

\begin{abstract}
Target detection and localization is one of the key research challenges in sensor networks. In this paper we propose a heterogeneous wireless sensor network integrating imaging and non-imaging sensors to accomplish the detection and localization task in complex urban environments. The lowcost non-imaging sensors provide early detection and partial localization of potential targets and direct imaging sensors to focus on them. Accurate target location estimated by the imaging sensors is subsequently used for in-situ calibration of the non-imaging sensors so that localization error is minimized over time. We evaluate our approach through simulation and our preliminary results reveal that coordination across different sensing modalities increases localization accuracy and can reduce the amount of imaging data that must be carried by the network.
\end{abstract}

\section{INTRODUCTION}

$\mathbf{T}$ HE ability to sense and interact with the physical world through networks of embedded wireless computers equipped with a multitude of sensors, enables a variety of applications not previously possible. Specifically, sensor network technology has been proposed for decentralized information processing tasks such as environmental monitoring [10], urban disaster recovery and rescue [11], sniper localization [14], as well as target identification and tracking [5], [6]. Furthermore, a number of large-scale deployments and demonstrations have shown that this technology can be successfully deployed in harsh environments with encouraging results.

These early successes motivate the use of Wireless Sensor Networks (WSNs) as persistent platforms for acquiring, processing, and transmitting surveillance information over large geographical areas. However, many challenges must be overcome before this technology is widely adopted and deployed. In this paper we outline an initial attempt to imbue adaptability to these sensor networks. We assert that this is a fundamental problem that must be addressed: sensor networks should be able to adapt and acquire information from previously unknown environments since sensors cannot be fully calibrated before deployment. This is especially true in urban environments where the environment is volatile and complex.

We propose a heterogeneous wireless sensor network that integrates imaging and non-imaging sensors to detect and localize targets in cluttered environments by adaptively estimating key environmental parameters, while performing the target localization task. In particular, we employ nonimaging sensors to estimate the coarse locations of targets, then utilize this information to guide imaging sensors to compute the target positions with higher precision. An optimization problem is formulated that identifies an optimal operating parameter and this information is fed back into the system to increase accuracy.

We validate the effectiveness of the proposed method via simulations. Initial results from these simulations indicate that the estimates of the non-imaging sensors' parameters converge to the real values even when the initial estimates are inaccurate and when (non-imaging) sensor readings contain considerable noise. Calibration quality increases with the number of independent sensor readings, but deteriorates when the level of camera noise is above a certain threshold. The reason behind this negative result is that when camera noise is high, feedback from the cameras is actually detrimental since it injects more uncertainty to the target localization problem.

The rest of the paper is organized as follows. We first motivate the use of heterogeneous sensor networks for target detection and localization in Section II and follow with a detailed presentation of the proposed localization and calibration algorithms in Section III. The proposed method is validated through simulations in a controlled environment; results from these simulations are summarized in Section IV. We present related work in Section V and conclude with a discussion on future extensions in Section VI. 


\section{Motivation}

Figure 1 illustrates a prototypical example of the proposed heterogeneous sensor network. The network consists of multiple cameras augmented by a set of non-imaging sensors (e.g. magnetometers, acoustic sensors, etc.) mounted on wireless sensor nodes embedded in the environment. The task of the system is to detect the presence and estimate the position of targets entering the sensor field (i.e. the geographic area covered by the network) using a combination of measurements from multiple nodes and multiple sensing modalities.

While target localization has received considerable coverage in the WSN literature (e.g., [5], [6], [14]), the novelty of the approach proposed here lies in the fact that it utilizes multiple sensing modalities to increase the accuracy of the provided target location estimates. The benefits gained by exploiting sensing diversity are primarily derived from using the strengths of one modality to compensate for the weaknesses of another. For example, imagery data is often preferred in military scenarios because it is easy to interpret and integrate with existing targeting and surveillance systems. Also, localization and tracking of identified targets can be performed accurately and efficiently. On the other hand, the use of cameras for unattended surveillance missions is limited by the significant power resources necessary to operate a surveillance camera, objects and structures occluding the cameras' fields of view, and the challenges of autonomous object recognition in cluttered environments. Fortunately, wireless sensor nodes provide an almost perfect complement to the weaknesses confronting camera networks. They are low cost, consume little power, and are easy to deploy; therefore they can extend the coverage of imaging sensors hampered by line-of-sight limitations. Furthermore, the use of additional sensing modalities can avoid the reliance on visible features for target identification and tracking. For example, a field of magnetometers can locate an unusually ferrous individual (person carrying a firearm), but would be unable to provide imagery for an operator to identify and classify the individual as a friend of foe.

Despite considerable improvements in their robustness and sensitivity, the current generation of miniaturized sensors remains rather faulty and crude. Even if the accuracy and robustness of such sensors is vastly improved, they need to be calibrated specifically for the environment where the network will be deployed. The insight of this paper is that sensing diversity can be exploited to improve the accuracy of the low cost sensor nodes. Achieving this goal will not only improve targeting performance to the point where the cameras are engaged rarely and only for high probability targets, but also extend the coverage of the camera network.

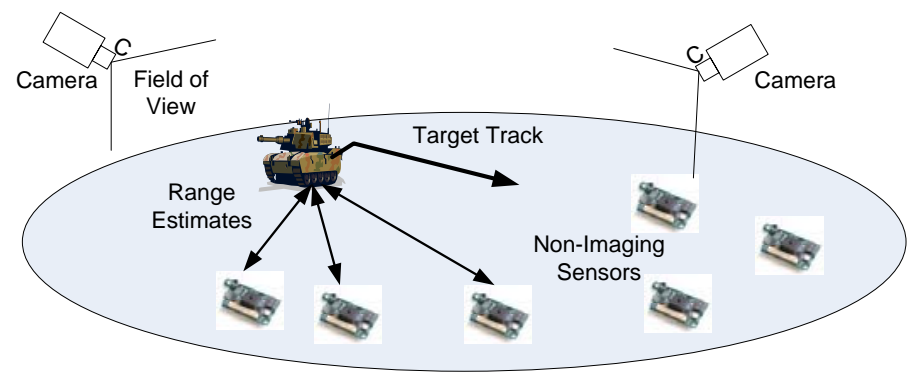

Fig. 1. Multi-modal sensor network for target tracking.

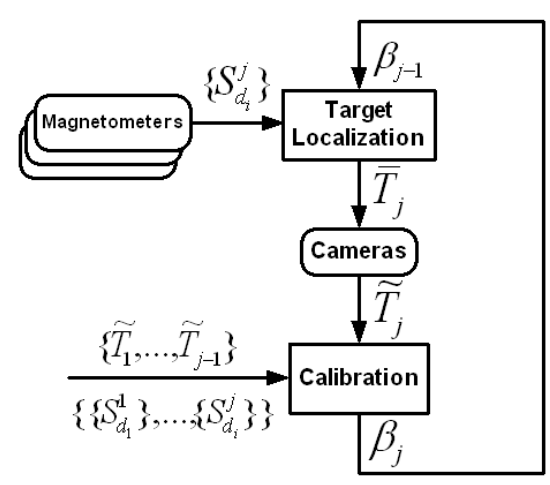

Fig. 2. Block diagram of the proposed target tracking system.

\section{DESIGN}

\section{A. Overview}

We present an approach, outlined in Figure 2, for in-situ calibration of a wireless sensor network of low-cost, nonimaging sensors with the help of a small number of highprecision imaging sensors. The calibration process occurs while the network is in operation, detecting and estimating the location of targets moving through the sensor field.

We assume that node $j$ in the sensor network can calculate its physical position $\left(x_{j}, y_{j}\right)$ either via a GPS receiver or by some other GPS-less technique (e.g. [3]). Furthermore, we assume that sensors can measure some form of energy energy emanating from the target (e.g. the target's magnetic field).

We denote the set of reported measurements as $\left\{S_{d_{i}}^{j}\right\}$, where $S_{d_{i}}^{j}$ is the measurement from sensor $s_{i}$ when the target is at location $T_{j}$. We assume that the signal strength measured at each sensor decays exponentially with an initially unknown decay exponent $\beta$ that is common for all non-imaging devices of the same modality. Given the current estimate $\beta_{j-1}$ of the decay exponent, signal strength measurements are translated to range estimates. Based on these range estimates, the estimated target location $\bar{T}_{j}$ is calculated through a collaborative trilateration algorithm. 
This location estimate is subsequently used to direct a set of pan-tilt-zoom cameras to "look" at the target. We assume that the cameras are pre-calibrated and can provide an independent estimate $\tilde{T}_{j}$ of the target location. This second location estimate is subsequently used to update the estimate $\beta_{j}$ of the decay exponent. This process repeats as the target moves, progressively providing more accurate estimates of the unknown parameter $\beta$ as well as the estimate of the target location $\bar{T}_{j}$ calculated by using only the non-imaging sensors.

In the remainder of this section we elaborate on the calibration and target localization mechanisms.

\section{B. Localization}

Sensor Model: We adopt the commonly-used, isotropic signal propagation model in which signal strength decreases according to a power $\beta>2$ of the distance from the source. This model has been used for localization algorithms that process acoustic ([1]) and magnetic signals ([12]).

The signal strength $S_{d}$ recorded at distance $d$ from the target is then given by:

$$
S_{d}=S_{T} / d^{\beta}+\mathcal{N}_{1},
$$

where $S_{T}$ is a constant representing the signal strength at distance zero and $\mathcal{N}_{1}$ represents an additive noise component that models the uncertainty introduced by the measurement mechanism. This measurement noise is modeled by a zero mean Gaussian random variable.

In our initial scenario and the simulations that follow, we assume that a nominal value of $S_{T}$ is known. Our model is parametrized by the decay exponent $\beta$ that is unknown and is primarily characterized by the local environment of the deployment area. The calibration problem is to iteratively refine the value of $\beta$ so that target localization is tailored to the deployment site.

Localization Strategy: Our initial approach to target localization using data from the the non-imaging sensors is given by solving a centralized linear least squares estimation problem. Given a value of $\beta$ and the sensor locations, a noisy range estimate from sensor $i$ to target $j$ can be obtained by inverting the sensor model from Eq.(1),

$$
\bar{d}_{i}^{j}=\left(\frac{S_{T}}{S_{d_{i}}^{j}}\right)^{1 / \beta}
$$

Then we can estimate the target location $\bar{T}_{j}=\left[\bar{X}_{T_{j}}, \bar{Y}_{T_{j}}\right]$ by solving a group of equations of the form:

$$
\left(x_{i}-\bar{X}_{T_{j}}\right)^{2}+\left(y_{i}-\bar{Y}_{T_{j}}\right)^{2}=\left(\bar{d}_{i}^{j}\right)^{2}, \quad i=1, \ldots, N,
$$

where $N$ is the number of sensors measuring the field of the target. With exact range data, $\bar{d}_{i}^{j}$, there will be a unique solution at the intersection of the circles defined by Eq.(3), provided that $N \geq 3$. When ranging data are noisy, an approximate solution is sought by linear least squares estimation.

For example, if there exist three sensors that detect the target, we attempt to solve the following equations for $\left[\bar{X}_{T_{j}}, \bar{Y}_{T_{j}}\right]$

$$
\begin{aligned}
\left(x_{1}-\bar{X}_{T_{j}}\right)^{2}+\left(y_{1}-\bar{Y}_{T_{j}}\right)^{2} & =\left(\bar{d}_{1}^{j}\right)^{2} \\
\left(x_{2}-\bar{X}_{T_{j}}\right)^{2}+\left(y_{2}-\bar{Y}_{T_{j}}\right)^{2} & =\left(\bar{d}_{2}^{j}\right)^{2} \\
\left(x_{3}-\bar{X}_{T_{j}}\right)^{2}+\left(y_{3}-\bar{Y}_{T_{j}}\right)^{2} & =\left(\bar{d}_{3}^{j}\right)^{2} .
\end{aligned}
$$

To avoid the non-linearity of the above equations, we can subtract any equation from another. For example, by subtracting Eq.(4) from Eq.(5), we obtain

$$
\begin{aligned}
2\left(x_{2}-x_{1}\right) \bar{X}_{T_{j}}+2\left(y_{2}-y_{1}\right) \bar{Y}_{T_{j}} & =\left(\left(\bar{d}_{1}^{j}\right)^{2}-x_{1}^{2}-y_{1}^{2}\right) \\
& -\left(\left(\bar{d}_{2}^{j}\right)^{2}-x_{2}^{2}-y_{2}^{2}\right) .
\end{aligned}
$$

Without loss of generality we have the following $N-1$ linear equations to solve for $\left[\bar{X}_{T_{j}}, \bar{Y}_{T_{j}}\right]$,

$$
\begin{aligned}
2\left(x_{i}-x_{1}\right) \bar{X}_{T_{j}}+ & 2\left(y_{i}-y_{1}\right) \bar{Y}_{T_{j}}=\left(\left(\bar{d}_{1}^{j}\right)^{2}-x_{1}^{2}-y_{1}^{2}\right) \\
& -\left(\left(\bar{d}_{i}^{j}\right)^{2}-x_{i}^{2}-y_{i}^{2}\right), \quad i=2, \ldots, N .
\end{aligned}
$$

Given noisy range data from $N \geq 3$ sensors, an approximate solution to the generally over-determined system of equations can be obtained by least squares estimation. In matrix notation, let $S$ denote the matrix of coefficients and $D$ denote a vector of the right hand sides of equations (8). Then a least squares estimate for

$$
S \bar{T}_{j}=D
$$

is given by minimizing the Euclidean norm squared of the residual $S \bar{T}_{j}-D$,

$$
\bar{T}_{j}^{*}=\operatorname{argmin}\left\|S \bar{T}_{j}-D\right\|^{2} .
$$

Thus given the current estimate of $\beta$, this centralized estimation problem localizes the target based on measurements from the non-imaging sensors. In this respect, this procedure also serves to indirectly evaluate the accuracy of the estimate of $\beta$ since the target location estimate it produces is compared with the target estimate provided by the cameras. The camera localization procedure will be discussed elsewhere. In this paper, we assume that the cameras are calibrated and have knowledge of the sensor locations and can therefore generate independent range estimates from each sensor to the target. We denote the camera-derived range estimate from sensor $i$ by $\tilde{d}_{i}$. 


\section{Calibration}

For each sensor detecting the target, a data pair $\left(\tilde{d}_{i}, S_{d_{i}}\right)$ is generated, where $S_{d_{i}}$ is the signal measurement obtained by sensor $i$ in the field and $\tilde{d}_{i}$ is the "true" range measurement produced by the cameras. One iteration of the calibration process yields an estimate of $\beta$ that minimizes the squared error over all sensors and target localization history. This is formulated as a nonlinear least squares optimization problem, with the cost function defined by

$$
\sum_{i, j}\left(S_{d_{i}}^{j}-S_{T} /\left(\tilde{d}_{i}^{j}\right)^{\beta}\right)^{2} .
$$

The value $\beta$ is estimated by performing gradient descent which yields a local minimum of the error function. The value of $\beta$ that minimizes the cost function is then used in the next iteration of the localization algorithm described above.

The proposed calibration mechanism can be applied to other target localization techniques as long as specific parameter(s), such as $\beta$ for the target localization technique considered here, are explicitly used in the localization algorithms. In general, the calibration problem can be formulated as the following optimization problem

$$
\operatorname{argmin}_{\theta} \sum_{j} \mathcal{C}\left(\bar{T}_{j}^{*}(\theta), \tilde{T}_{j}\right)
$$

where $\theta$ is the parameter(s) required in arriving at the target location estimate $\bar{T}_{j}^{*}$ and $\mathcal{C}(\cdot, \cdot)$ is a metric that measures the discrepancy between the estimates obtained from different sensor modalities (non-imaging sensors versus cameras).

\section{Evaluation}

As the first step in our evaluation process, we implemented the proposed algorithms in Matlab. In this way we can validate the efficacy of our approach in a controlled simulation environment before we test it under realistic settings.

To simulate the parameter estimation process with multimodal calibration, we assume a single target moves with a stable speed along a straight line on the $X-Y$ plane. At each location, the target is within detection range of $N$ sensor nodes that are randomly located over the same plane. Furthermore, we assume that $S_{T}$ is known and the clocks of the nodes in the sensor network are synchronized. Finally, each sensor node knows its own position and the measured signal strength at each sensor follows Eq. 1.

Simulation under controlled conditions enables us to validate our method qualitatively and at the same time evaluate its performance quantitatively. Our high-level goal is to investigate how feedback from the cameras can minimize or dilate the localization error from low-cost non-imaging sensors under various deployment scenarios. We focus on two quantitative metrics:

1) Convergence speed, in terms of the number of measurements to estimate $\beta$ and the resulting estimation accuracy $|\beta-\bar{\beta}|$

2) Localization errors $\|T-\bar{T}\|$ and $\|\tilde{T}-\bar{T}\|$.

We study the impact of initial value $\beta_{0}$, group size $N$, non-imaging sensing noise $\mathcal{N}_{1}$, and camera observation noise $\mathcal{N}_{2}$ on the two metrics above. Signal noise $\mathcal{N}_{1}$ is modeled as a Gaussian random variable with zero mean and standard deviation that is a constant percentage $\left(r_{s}\right)$ of the signal strength at distance zero. In our simulations we choose $r_{s}=5 \%, 10 \%, 15 \%, 20 \%$. Finally, we set the standard deviation of $\mathcal{N}_{2}$ to be equal to $3 \%, 4 \%, \ldots, 10 \%$ of the actual distance $d_{j}$ between sensor $j$ and the target.

\section{A. Influence of initial value $\beta_{0}$}

To evaluate the influence of the initial input $\beta_{0}$ on the non-linear least square fitting of $\bar{\beta}$, we choose $\beta_{0}=1,3,5$ ,7, 9. Figure 3.(a) plots the difference between the estimated $\bar{\beta}$ and the actual $\beta$ (set to 3.471 based on readings from an actual magnetometer) over a number of measurements when $r_{s}=10 \%$ and $N=8$. As the figure indicates, $\bar{\beta}$ always converges to the same value regardless of the initial value of $\beta_{0}$. For the convergence of $\bar{T}$ to $T$, similar results under different $\beta_{0}$ 's are illustrated in Figure 3.(b). These results are encouraging since they indicate that the $\beta$ estimate converges in a small number of measurements and localization error quickly diminishes. The same results hold when $r_{s}=5 \%, 15 \%, 20 \%$, not presented here in the interest of space. For the remainder of our evaluation we set $\beta_{0}$ to 3 .

\section{B. Effect of group size $N$}

Figure 4.(a) illustrates the difference of the estimated $\bar{\beta}$ and real $\beta$ over the number of measurements for different group size $N$ when $\mathcal{N}_{1}=\left(0,0.05 S_{T}\right)$ and $\mathcal{N}_{2}=(0,0)$. Figure 4.(b) plots the corresponding location error. It is evident from these results the $\beta$ estimation improves as group size increases however even small groups of sensors can achieve good $\beta$ estimates. The intermittent localization error apparent in Fig. 4 in the case of small groups is due to the random noise $\mathcal{N}_{1}$, whose effect is canceled when $N$ increases. 


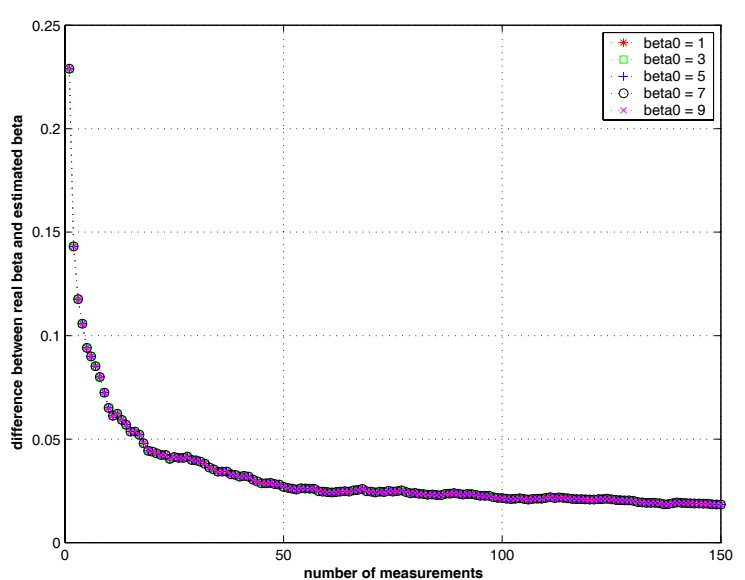

(a) $|\beta-\bar{\beta}|$ when $\mathcal{N}_{1}=\left(0,0.10 S_{T}\right)$, $\mathcal{N}_{2}=(0,0)$ and $N=8$

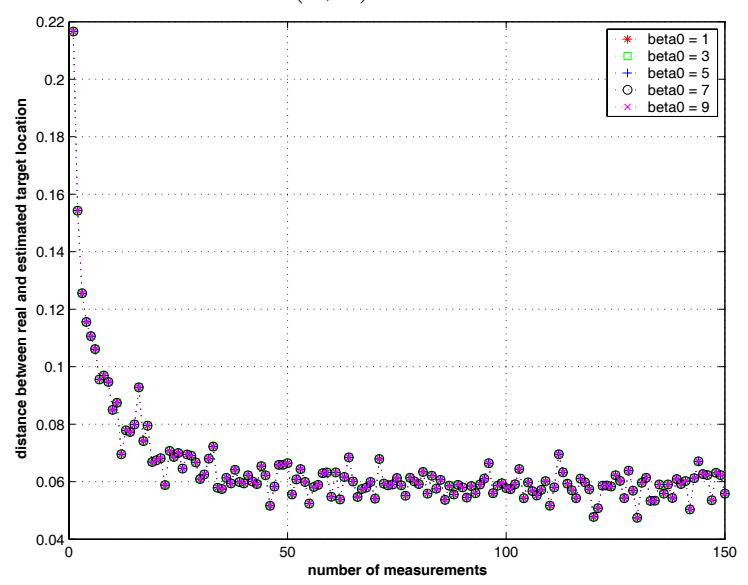

(b) $\|T-\bar{T}\|$ when $\mathcal{N}_{1}=\left(0,0.10 S_{T}\right)$, $\mathcal{N}_{2}=(0,0)$ and $N=8$

Fig. 3. Estimates for $\bar{\beta}$ and $\bar{T}$ versus the number of measurements for different $\beta_{0}$ when $\mathcal{N}_{1}=\left(0,0.10 S_{T}\right), \mathcal{N}_{2}=(0,0)$ and $N=8$.

\section{Influence of signal noise $\mathcal{N}_{1}$ and camera localization noise $\mathcal{N}_{2}$}

To study the influence of signal noise $\mathcal{N}_{1}$ to the localization error, we perform the same experiment with different $\mathcal{N}_{1}$ settings. Figure 5 presents the $|\beta-\bar{\beta}|$ error as well as the $\|T-\bar{T}\|,\|\tilde{T}-\bar{T}\|$ errors for different combinations of signal noise $\mathcal{N}_{1}$ and camera localization noise $\mathcal{N}_{2}$. Under three groups of $\mathcal{N}_{2}$ settings, the errors converge to values that are in proportion to $\mathcal{N}_{2}$ 's value. Notice however that the errors are insensitive to $\mathcal{N}_{1} \mathrm{~s}$. Therefore, we can conclude that the calibration errors for $\beta$ and target localization errors are mainly contributed by the camera noise $\mathcal{N}_{2}$. Intuitively this means that when the camera noise $\mathcal{N}_{2}$ is large, feedback from the cameras is detrimental to the calibration process since the camera range estimates are worse than the range estimates provided the non-imaging sensors even when inaccurate $\beta$ values are used.

We confirm this intuition by varying the camera noise

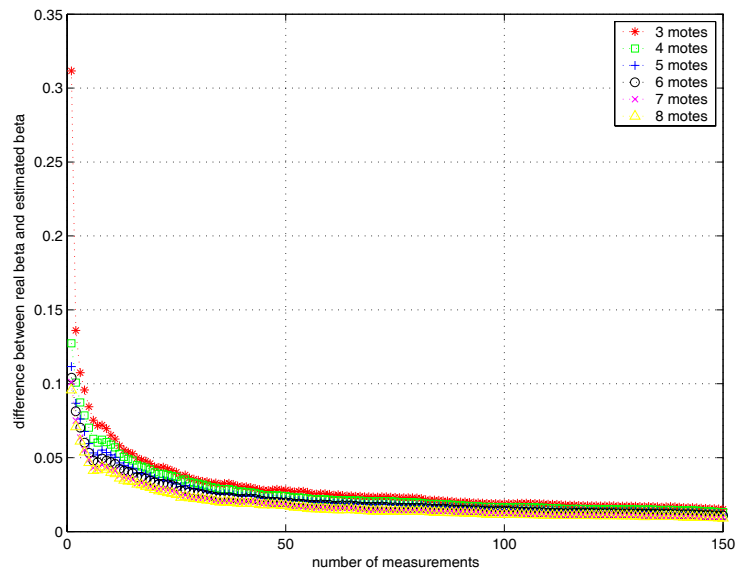

(a) $|\beta-\bar{\beta}|$ when $\mathcal{N}_{1}=\left(0,0.05 S_{T}\right)$ and $\mathcal{N}_{2}=(0,0)$

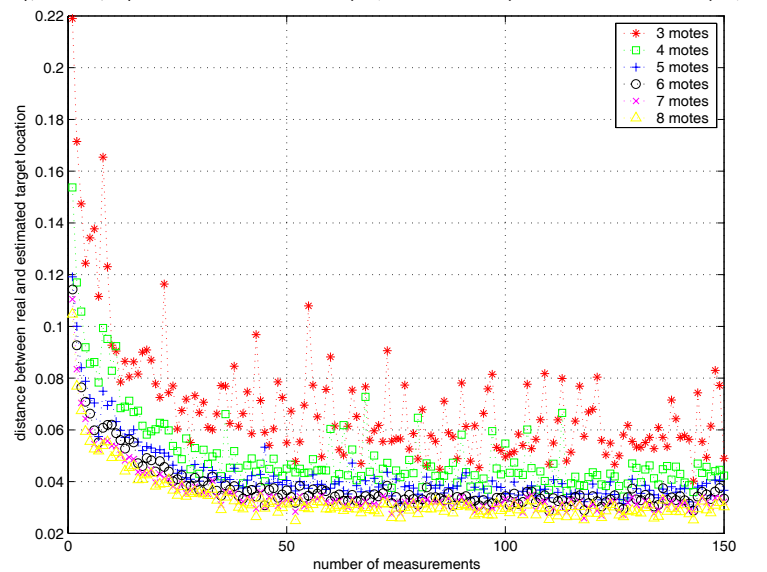

(b) $\|T-\bar{T}\|$ when $\mathcal{N}_{1}=\left(0,0.05 S_{T}\right)$ and $\mathcal{N}_{2}=(0,0)$

Fig. 4. Calibration for $\bar{\beta}$ and $\bar{T}$ versus the number of measurements for different group size $N$ when $\mathcal{N}_{1}=\left(0,0.05 S_{T}\right)$ and $\mathcal{N}_{2}=(0,0)$.

while keep the other parameters constant. Figure 6 illustrates the calibration results for different camera localization noises $\mathcal{N}_{2}$ when $\mathcal{N}_{1}=\left(0,0.05 S_{T}\right)$ and $N=8$. It is clear that both $\bar{\beta}$ error and $\bar{T}$ error increase with larger $\mathcal{N}_{2}$ to a point after which feedback from the cameras is actually detrimental to the calibration process.

\section{RELATED WORK}

If sensors are to provide accurate tracking results, they must be calibrated to the environments in which they will be deployed. Previous work has investigated the problem of global calibration of large-scale, densely-deployed sensor network using a two-phase collaborative approach [2] or a parameter estimation that optimizes the overall system performance [15]. In this paper, the non-imaging sensor devices are calibrated based on target estimates from the imaging sensors. This forms a collaborative, heterogeneous sensor network for target detection and localization.

Zou and Chakrabarty [18] proposed an energy-aware 
target detection and localization strategy for cluster-based wireless networks. Rabbat et al. [13] present a robust localization algorithm of an isotropic energy source using kernel averages of measurements from distributed sensors. Blatt and Hero [1] describe the aggregated projection onto convex sets (APOCS) method by treating the localization problem in its convex feasibility formulation. Li et al. [7]

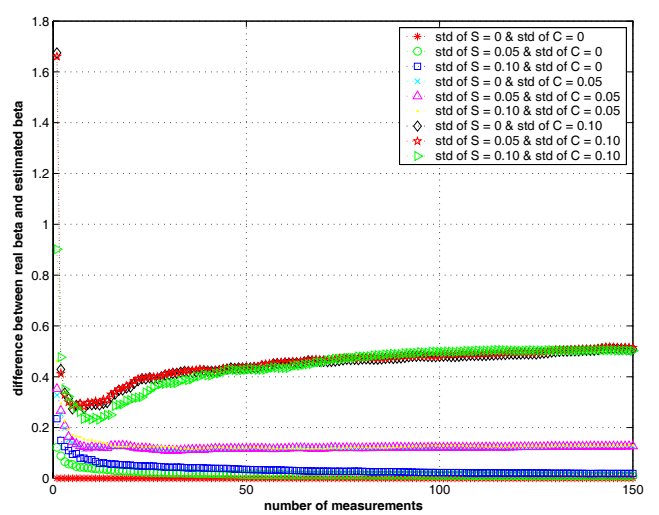

(a) $|\beta-\bar{\beta}|$ for different $\mathcal{N}_{1}$ and $\mathcal{N}_{2}$ when $N=8$

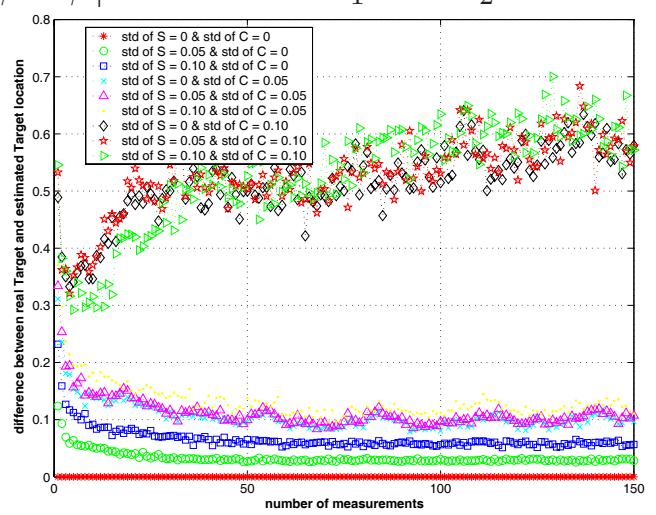

(b) $\|T-\bar{T}\|$ for different $\mathcal{N}_{1}$ and $\mathcal{N}_{2}$ when $N=8$

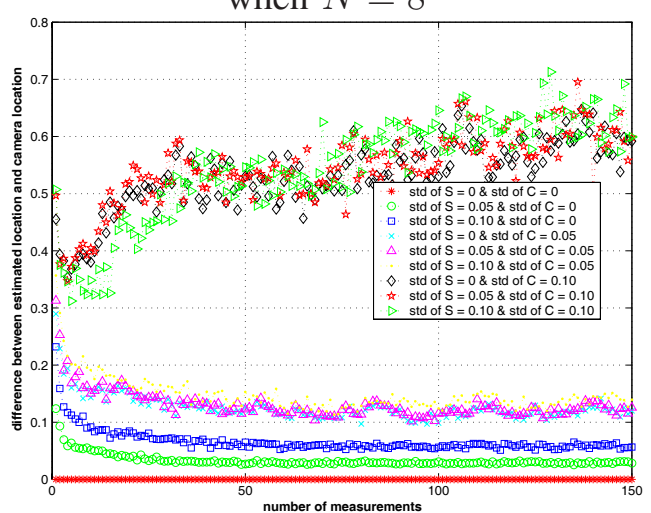

(c) $\|\tilde{T}-\bar{T}\|$ for different $\mathcal{N}_{1}$ and $\mathcal{N}_{2}$ when $N=8$

Fig. 5. Illustration of the calibration errors, $|\beta-\bar{\beta}|,\|T-\bar{T}\|$ and $\|\tilde{T}-\bar{T}\|$, of $\bar{\beta}$ and $\bar{T}$ versus the number of measurements for different combinations of $\mathcal{N}_{1}$ and $\mathcal{N}_{2}$ when $N=8$. estimate target position by solving a non-linear least squares problem and assuming that sensors are pre-calibrated. Target localization based on time-of-arrival (TOA) [4] or the direction-of-arrival (DOA) of acoustical/seismic signals has also been explored in the past [16]. In [17], a spanning tree rooted at the sensor node close to a target is used

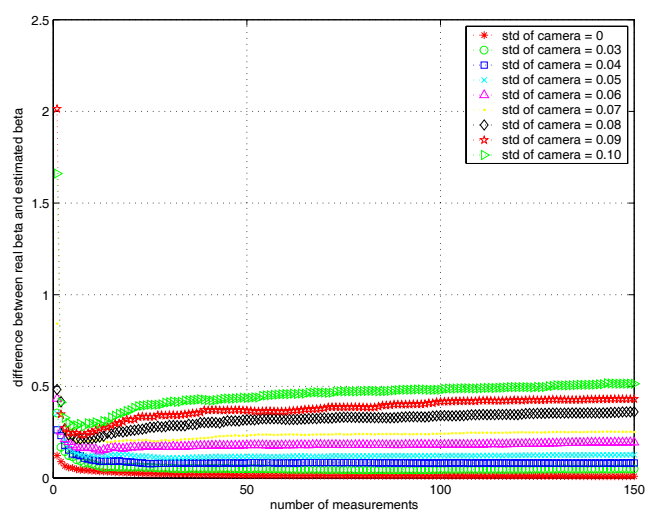

(a) $|\beta-\bar{\beta}|$ for different $\mathcal{N}_{2}$ when $\mathcal{N}_{1}=\left(0,0.05 S_{T}\right)$

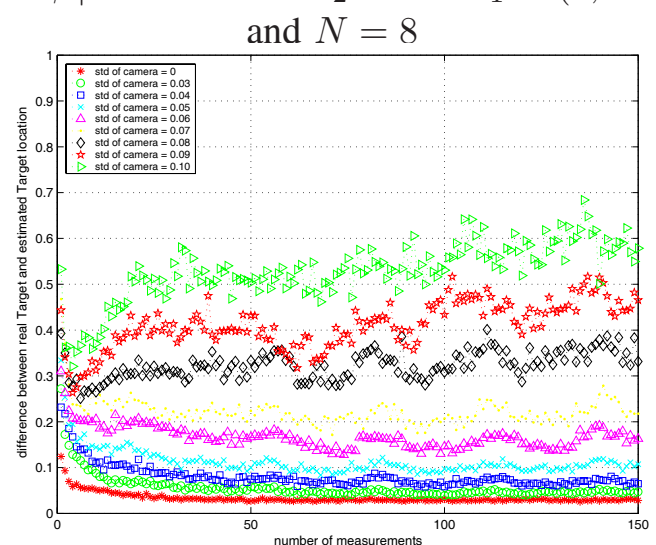

(b) $\|T-\bar{T}\|$ for different $\mathcal{N}_{2}$ when $\mathcal{N}_{1}=\left(0,0.05 S_{T}\right)$

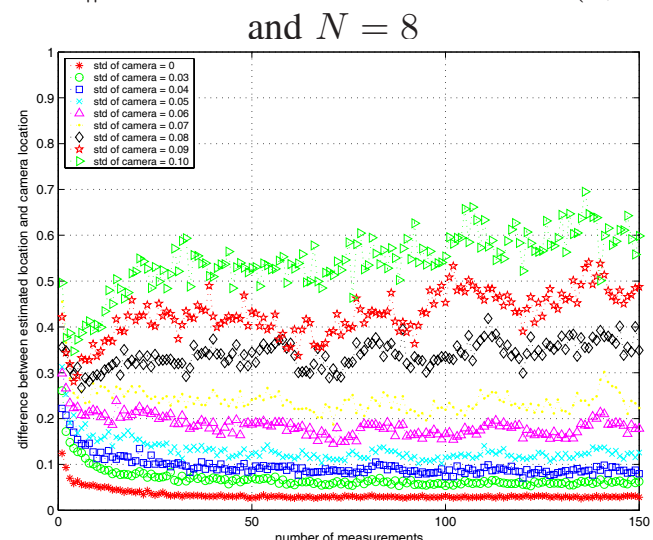

(c) $\|\tilde{T}-\bar{T}\|$ for different $\mathcal{N}_{2}$ when $\mathcal{N}_{1}=\left(0,0.05 S_{T}\right)$ and $N=8$

Fig. 6. Illustration of the calibration errors, $|\beta-\bar{\beta}|,\|T-\bar{T}\|$ and $\|\tilde{T}-\bar{T}\|$, of $\bar{\beta}$ and $\bar{T}$ versus the number of measurements for different $\mathcal{N}_{2}$ when $\mathcal{N}_{1}=\left(0,0.05 S_{T}\right)$ and $N=8$. 
for target tracking, with target position estimated by the location of the root sensor. In this paper, we focus on leveraging multi-modal sensor networks comprising both low-cost, non-imaging sensors and direct image sensors to calibrate the measurements from the non-imaging sensors, while concurrently localizing targets as they transit through the sensor field.

\section{Vi. Conclusions And Future Work}

We present a method to calibrate non-imaging sensors used in target tracking applications through imaging sensors. Specifically, non-imaging sensors provide an initial (inaccurate) location of the target and task the cameras to identify the target. Based on the provided information, one or more cameras provide an independent estimation about the target's actual location. The estimated target location provided by the cameras is subsequently used to adjust the exponent $\beta$ of the signal propagation model used by the non-imaging sensors.

Our preliminary simulation results show that the proposed mechanism can estimate the correct value of $\beta$ in a few iterations even when the original estimate is very inaccurate. Only when the location estimate provided by the cameras is very inaccurate (i.e. $\mathcal{N}_{2}$ is large) $\beta$ estimates and localization errors diverge.

As part of our future plans we will implement and evaluate the proposed approach on a sensor network testbed that uses MicaZ motes [8] equipped with MTS 310 magnetometers [9] and a camera to track ferrous targets.

\section{REFERENCES}

[1] D. Blatt and A.O. Hero, APOCS: A Rapidly Convergent Source Localization Algorithm for Sensor Networks, In Proceedings of IEEE Workshop on Statistical Signal Processing (SSP), Bordeaux, July 2005

[2] V. Bychkovskiy, S. Megerian, D. Estrin, and M. Potkonjak, A Collaborative Approach to In-Place Sensor Calibration, In Proceedings of IPSN, 2003.

[3] X. Cheng, A. Thaeler, G. Xue, and D. Chen, TPS: a time-based positioning scheme for outdoor sensor networks, In Proceedings of INFOCOM, 2004.

[4] D. Friedlander, C. Griffin, N. Jacobson, S. Phoha, and R. R. Brooks, Dynamic Agent Classification and Tracking Using an Ad Hoc Mobile Acoustic Sensor Network, Eurasip Journal on Applied Signal Processing, 2003:4 (2003) 371-377.

[5] Lin Gu, Dong Jia, Pascal Vicaire, Ting Yan, Liqian Luo, Aajay Tirumala, Qing Cao, Tian He, John A. Stankovic, Tarek Abdelzaher, and Bruce Krogh, Lightweight Detection and Classification for Wireless Sensor Networks in Realistic Environments, In Proceedings of the Third ACM Conference on Embedded Networked Sensor Systems (SenSys 2005), November 2005.

[6] Tian He, Suda Krishnamurthy, John A. Stankovic, Tarek F. Abdelzaher, Liqian Luo, Radu Stoleru, Ting Yan, Lin Gu, Jonathan Hui, and Bruce Krogh, An Energy-Efficient Surveillance System Using Wireless Sensor Networks, In Proceedings of the Second International Conference on Mobile Systems, Applications, and Services (MobiSys), June 2004.
[7] D. Li, K.D. Wong, Y.H. Hu, and A.M. Sayeed, Detection, Classification, and Tracking of Targets, IEEE Signal Processing Magazine, Vol. 19, pp. 17-29, March 2002.

[8] http://www.xbow.com/products/productsdetails. aspx?sid=101

[9] http: //www.xbow. com/products/productsdetails. aspx?sid=75

[10] Răzvan Musăloiu-E., Andreas Terzis, Katalin Szlavecz, Alex Szalay and Joshua Cogan, Jim Gray, Life Under your Feet: A Wireless Sensor Network for Soil Ecology, In Proceedings of the $3^{\text {rd }}$ EmNets Workshop, May 2006.

[11] David Malan, Thaddeus Fulford-Jones, Matt Welsh, Steve Moulton, CodeBlue: An Ad Hoc Sensor Network Infrastructure for Emergency Medical Care, In Proceedings of the MobiSys 2004 Workshop on Applications of Mobile Embedded Systems (WAMES 2004), June 2004.

[12] Songhwai Oh, Luca Schenato, Phoebus Chen, and Shankar Sastry, A Scalable Real-Time Multiple-Target Tracking Algorithm for Sensor Networks. Technical Report UCB//ERL M05/9, University of California, Berkeley, February 2005.

[13] M. Rabbat, R. Nowak, and J. Bucklew, Robust decentralized source localization via averaging, In Proceedings of the IEEE International Conference on Acoustics, Speech, and Signal Processing (ICASSP), 2005.

[14] Gyula Simon, Miklos Maroti, Akos Ledeczi, Gyorgy Balogh, Branislav Kusy, Andreas Nadas, Gabor Pap, Janos Sallai, Ken Frampton Sensor Network-Base Countersniper System In Proceedings of SenSys 2004, November 2004.

[15] K. Whitehouse and D. Culler, Macro-calibration in Sensor/Actuator Networks, Mobile Networks and Applications Journal (MONET), Special Issue on Wireless Sensor Networks, June, 2003.

[16] L. Yip, K. Comanor, J. C. Chen, R. E. Hudson, K. Yao, and L. Vandenberghe, Array Processing for Target DOA, Localization, and Classification based on AML and SVM Algorithms in Sensor Networks, In Proceedings of the 2nd International Workshop on Information Processing in Sensor Networks (IPSN'03), 2003, Palo Alto, California, USA.

[17] W. Zhang and G. Cao, Optimizing Tree Reconfigration for Mobile Target Tracking in Sensor Networks, In Proceedings of IEEE INFOCOM, March 2004.

[18] Y. Zou, Krishnendu Chakrabarty, Sensor Deployment and Target Localization in Distributed Sensor Networks, ACM Transactions on Embedded Computing Systems, Vol. 3, No. 1, February 2004, Pages 61-91. 